\title{
Indagine critica sui modelli di atmosfera $\left(^{*}\right)$
}

\author{
G. I)'ELIA (**)
}

Ricevuto il 18 Maggio 1963

Riassunto. - Nella prima parte del lavoro e individuato il modello piu attendibile di atmosfera, che deve dunque essere adottato nei calcoli relativi a problemi aeronautici e missilistici.

Nella seconda parte vengono seritte le equazioni dell'atmosfera considerata come campo fluidodinamico costituito da piu componenti elettricamente conduttivi e reagenti. Con una serie di successive approssimazioni sono derivate le equazioni semplificate che attualmente sono usate per il calcolo delle tabelle dell'atmosfera; da un sistema complesso di equazioni, ma determinato matematicamente, si ottiene un sistema semplice, ma doppiamente indeterminato; alla inderminatezza si deve sopperire con sondaggi sperimentali.

Nell'ultima parte del lavoro si fa una critica alla base di calcolo del piu attendibile modello, rilevando tra l'altro che esso e relativo alle condizioni di un punto singolare (Fort (hurchill, nel Canada) posto in prossimita del polo magnetico.

SUMARY. - - The first part of the paper is devoted to determine the more reliable model for the atmosphere that should be used in computations related with aeronantical and missilistic prohlems.

In a second part the equations are earried out for the atmosphere considered as a fluid-drnamical field constituted by reacting and electrically conductive components. The simplified equations used up to day for the computations of the atmospheric tables are derived through a series of approximations: from a complex set of equations, mathematically determined,

(*) Nota presentata al 20 Congresso Internazionale Tecnico scientifico dello Spazio. Roma, 19-23 Giugno 1962.

$\left({ }^{* *}\right)$ Universita di Napoli - Istituto di Aerodinamica. 
a twice undetermined simple system is obtained; esperimental soundings must supply to the undeterminations.

In the final part of this work, the critic is devoted to the more reliable model, by enhancing also that it is connected with the conditions in a sin. gular point (Fort Churchill-Canada) located close to the magnetic pole.

\section{I - INTRODUZIONE.}

I progressi della propulsione a getto e la programmazione di missioni spaziali, hanno posto il problema della esplorazione dello spazio intorno alla Terra.

Sono richieste informazioni sulle radiazioni provenienti dagli spazi esterni, per la protezione dell'equipaggio umano (il problema si presenta gia nel volo convenzionale, cioe con propulsione chimica e sostentazione aerodinamica, alle alte quote; sara pii grave fuori l'atmosfera); informazioni sulle onde a frequenza radio provenienti dal sole (infatti onde di tale frequenza possono arrecare, per interferenza, disturbi nella guida a distanza dei missili) e numerose altre informazioni.

Le ricerche sulla atmosfera terrestre rappresentano un settore della vasta indagine attualmente in pieno sviluppo.

Tutti i problemi di interazione tra missile e ambiente (di progettazione della forma e della struttura del missile, di progettazione del propulsore, della protezione, al rientro, dal riscaldamento aerodinamico) richiedono, per l'ingegnere missilistico, la disponibilita di dati numerici delle grandezze che intervengono nelle leggi di interazione.

Tali dati sono raccolti in tabelle, frutto della ricerca teorica e di determinazioni sperimentali.

I dati sono ottenuti attraverso la integrazione di equazioni che interpretano l'atmosfera come campo fluido. I dati ottenuti col calcolo relativi alle zone inferiori (fino a circa $32 \mathrm{~km}$ ) sono verificati con numerosi sondaggi diretti; la zona successiva (fino a circa $200 \mathrm{~km}$ ) è definita "speculativa ", per essa manca un numero sufficiente di dati sperimentali, la zona superiore (fino a circa $1000 \mathrm{~km}$ ) è definita di "tentativon, cioe deve essere ritenuta come pura extrapolazione delle zone inferiori.

U.S.A. e U.R.R.S. hanno fornito la parte piu grande delle informazioni sull'atmosfera; Paesi minori si associano progressivamente ai programmi di ricerca.

In U.S.A. l'approntamento delle tabelle e affidato a gruppi di lavoro presso le Universita, i quali dipendono dai comandi militari (Army, 
Navy, Air Force) e sono coordinati a livello superiore dal dipartimento della difesa (Defende Department Interservice Coordinating Group).

Attualmente si dispone di numerose tabelle, le quali, purtroppo, spesso vengono usate indifferentemente; di conseguenza soluzioni diverse di un problema operazionale ugualmente posto, possono risultare " non confrontabili ". Inoltre, le tabelle, vengono usate ignorando il grado di attendibilita dei dati e le limitazioni accettate per giungere in modo semplice alla loro compilazione.

Gli obbiettivi della nostra nota sono:

— individuare le tabelle attualmente piu attendibili che l'ingegnere missilistico deve usare,

- derivare il sistema di equazioni che regge il campo fluidodinamico atmosferico, reale. Descrivere, successivamente, le posizioni accettate per giungere al sistema di equazioni semplice, usato per le tabulazioni,

- esaminare i metodi di indagine sperimentale,

- fare una analisi critica della base di calcolo attualmente adot$\operatorname{tata}\left({ }^{*}\right)$.

\section{II - I MODELII DI ATMOSFERA.}

Il primo passo, al fine di ottenere una specificazione dettagliata dell'atmosfera, veniva da parte della organizzazione internazionale dell'aviazione civile (ICAO), la quale elaborava nel 1952 un progetto di atmosfera.

Alla iniziativa si associavano diversi Stati tra cui l'Italia, nel 1954 compare una edizione ICAO Italiana $\left({ }^{1}\right)\left({ }^{* *}\right)$. Parallelamente, sempre nel 1954, vennero pubblicate due altre edizioni ICAO; una a Montreal (Canada) $\left({ }^{2}\right)$ e una a Washington (USA) $\left(^{3}\right)$. Successivamente, nel 1955 ,

(*) Ringrazio il prof. Napolitano per i suggerimenti utili all'impostazione del lavoro. Ringrazio il prof. Col. Fea per avere permesso l'esame della bibliografia sull'atmosfera, raccolta presso il Yinistero dell'Aeronautica dal sottotenente dott. Iuccio della Divisione Studi e il dott. Iuccio per le produttive discussioni.

(**) I'edizione fu curata dalla " Direzione Generale dell'A viazione Civile del Traffico Aereo " del Ministero della Difesa Aeronantica con la collaborazione dell'Istituto per applicazioni di calcolo del Consiglio Nazionale delle Ricerche. 
veniva preparato, a cura della NACA (National Committee for Aeronautic) la pubblicazione "Standard Atmosphere " ( $\left.{ }^{4}\right)$.

L'esigenza di avere un miglioramento ed una estensione dei dati cra ormai avvertita ed in rapida successione si approntavano le tabelle "Extension to the ICAO Standard Atmosphere" ( $\left.{ }^{5}\right)$, e " $\triangle$ RDC Model Atmosphere 1956 " dell'Air Force Survey in Geophysies " ( $\left.{ }^{6}\right)(*)$.

Tutte le pubblicazioni rappresentano il conseguente sviluppo di una medesima impostazione di alcolo delle proprieta, dunque sono fortemente dipendenti ed ognuna e il successivo progresso della precedente. Si deve far notare subito, che esistono, per alcune zone di atmosfera comuni nelle successive pubblicazioni, rilevanti diversita dei valori numerici.

Il modello ARDC $1956\left({ }^{6}\right)$, e coerente con i precedenti modelli (ICAO e ICAO-NACA) tra il livello del mare e $20 \mathrm{~km}$ di quota $(65.800$ feets) $\left(^{8}\right)$; tra $20 \mathrm{~km}$ e $300 \mathrm{~km}$ (984.252 feets) e coerente con le tabelle "Extension to the ICAO Standard Atmosphere" $\left({ }^{8}\right)$.

Il modello ARDC 1956 fornisce dati numerici fino alla quota di $500 \mathrm{~km}$ (1.604.420 piedi) ed e basato su dati sperimentali fino a $120 \mathrm{~km}$ (400.000 piedi); solo pochissimi dati sono disponibili per la regione tra 120 e $220 \mathrm{~km}$ e nessun rilevamento diretto esiste per le zone superiori $\left.{ }^{8}\right)$. Di conseguenza, il modello diviene poco attendibile, per quote superiori a $100 \mathrm{~km}\left(^{8}\right)$. La zona tra 220 e $500 \mathrm{~km}$ di questo modello e essenzialmente calcolata con posizioni teoriche; tra i diversi indirizzi teorici, delle diverse scuole, e stato assunto, per la preparazione di questo modello, quello che dava piu severe condizioni di resistenza aerodinamiea e riscaldamento aerodinamico $\left(^{8}\right)$.

È chiaro che le deficienze e le limitazioni del modello $A R D C 1956$ ponevano il problema della sua verifica e correzione oltre che quello della estensione. A cio prorvedeva, per quanto e stato possibile, il successivo, piu recente e piu completo modello ARDC 1959.

In esso confluiscono praticamente gli sforzi di indagine di tutti i gruppi americani di ricerca e i contributi di Universita americane e non americane, sotto gri auspici della "Air Research and Development, Command ". Vengono effettuate correzioni dei dati teorici del precedente modello alla luee dei dati racoolti direttamente dai razzi e dei dati

$(*)$ A cura del "Centro Studi Propulsione a Reazione" sono state calcolate, in Italia, dall'Istituto Nazionale per le applicazioni di calcolo del C.N.R., aleune grandezze caratteristiche della atmosfera tipo "ARDC $1956 »\left({ }^{7}\right)$. 
di densita calcolati con la osservazione delle variazioni dellorbita dei satelliti artificiali americani e russi.

Jat storia dei modelli di atmosfera che brevemente abbiamo riassunta, ci autorizza a localizzare il nostro studio al modello ARDC 1959 che corregge ed amplia il modello ARDC 1956.

Il modello ARDC 1959 e dunque il piu recente di cui si dispone ed e anche il piu esteso (fornisce dati fino a 700.000 metri e tenta la estrapolazione di essi fino ad 1 milione di metri).

Nello stesso tempo e il piu attendibile, perehe è conseguente ai precedenti modelli ed e basato sulla verifica (o correzione, attraverso le informazioni dei sondaggi diretti dell'atmosfera), dei dati numerici degli altri modelli.

\section{Elenco dei simboli}

$B \quad=$ intensita del flusso di induzione magnetica

$D \quad=$ flusso di induzione elettrostatica

$D_{i j}=$ coefficiente di diffusione

$E=$ intensita del ampo elettrico

$\mathrm{F}_{\mathrm{cst}}=$ forza esterna (per unita di massa)

$y$ - campo gravitazionale

$H$. = vettore forza magnetica

$H^{\prime}=$ quota geopotenziale

$i=$ densita della corrente di induzione

$J_{i} \quad$ - flusso microscopico di massa

$J_{q}=$ flusso di calore

$\mathrm{m}^{\prime}=$ unita geopotenziale (metro)

$M=$ peso molecolare medio

$M_{i}=$ peso molecolare del componente $\mathrm{i}^{\mathrm{mo}}$

$p=$ pressione

$P^{+}=P_{1}+P_{2}+P_{3}=$ tensore degli sforzi superficiali

$P_{k}(k=1,2,3)=$ rispettivamente tensori degli sforzi normali, viscosi, elettromannetici

$P=$ "proprieta" generica del campo

$\dot{q}_{1}, \dot{q}_{2}=$ produzione di energia sotto forma di calore

$r \quad=$ direzione generica nel rampo fluido

$R^{+}=$costante universale dei gas

$t$ variabile tempo

$t_{\text {ch }}=$ variabile tempo ehimico

$T=$ temperatura 
$T_{M}=$ temperatura in scala molecolare

$v=$ velocita mecroscopica media di massa

$Z \quad=$ quota geometrica

$\varepsilon=$ costante dielettrica

$\mu \quad=$ coefficiente di viscosita

$\lambda=$ coefficiente di conducibilita termica

$\mu^{+}=$permeabilita magnetica

$\varrho=\sum_{1}^{n} \varrho i=$ densita di massa

$\varrho_{i}=$ densita del componente iesimo

$\varrho_{i o}=\frac{\varrho_{i}}{\rho}=$ concentrazione del componente imo

$\dot{\varrho}_{i}=\frac{\partial}{\partial t_{c h}} \underline{o}_{i}=$ produzione del costituente imo

$\varrho_{e}=$ densita delle cariche elettriche

$\sigma=$ conducibilita elettrica

$\tau=$ sforzo viscoso

Operatori.

$X \quad=$ indica "prodotto scalare"

$\Lambda$ = indica "prodotto vettoriale "

$\frac{\partial}{\partial r}=\frac{\partial}{\partial x} i+\frac{\partial}{\partial u} \bar{\jmath}+\frac{\partial}{\partial r} \bar{k}$ operatore gradiente

$i, \overline{\mathrm{j}}, l_{i}=$ versori degli assi $x, y, z$.

III - Studio DELL'ATMOSFERA COME CAMPo FLUIdodinamico.

1, III. - Fenomeni nell'atmosfera.

L'atmosfera terrestre è investita da flussi di energia e di massa, l'intensita dei quali è in correlazione con l'attivita solare; gli effetti di questo trasferimento di energia e di massa si manifestano con variazioni delle proprieta della alta atmosfera.

Radiazioni del tipo radio $(3-30 \mathrm{~cm})$, ultraviolette e $\mathrm{X}$ sono emesse in vicinanza delle macchie solari ed assorbite dall'atmosfera specialmente alle altitudini comprese tra 150 e $200 \mathrm{~km}$. La loro energia è sufficiente a riscaldare l'atmosfera causando una espansione verso l'alto ed una variazione della densita dell'aria ( $\left.{ }^{9}\right)$. La correlazione tra attivita solare e densita dell'atmosfera spiega le fluttuazioni impreviste della 
resistenza di alcuni satelliti nel loro moto orbitale (studi di Jacchia, Prister $\left.\left({ }^{\theta}\right)\right)$.

Oltre il flusso di radiazioni, nubi di particelle fortemente energetiche in partenza dalle fiammate solari provocano effetti geofisici. Isa formazione delle fasce di Van Allen è spiegata dalla cattura delle nubi di plasma solare da parte del campo magnetico terrestre. Le fascie Van Allen immagazzinano massa ed energia proveniente dal sole (ad altitudini tra 2000 e $100.000 \mathrm{~km}$ ); in opportune condizioni di perturbazione le particelle energetiche deviano dalla loro traiettoria a spirale, intorno alle linee di forza del campo magnetico e trasferiscono energia cinetica all'atmosfera mediante collisioni ionizzanti.

Inoltre si verifica un flusso di massa inverso, nella direzione atmosfera-spazio esterno. Alcune particelle, nelle zone estremamente rarefatte della atmosfera, dopo l'ultima collisione, possono avere acquistato sufficiente energia (energia di sfuggita del campo gravitazionale), da seguire una traiettoria aperta iperbolica.

L'atmosfera può dunque essere considerata eampo fluidodinamico attraversato da flussi di massa nei due sensi, e da flussi di radiazione provenienti dal sole, che sono causa di fenomeni geofisici, quali trasformazioni chimiche, produzione e decadimento di elettroni, tempeste magnetiche, aurore, venti; tali fenomeni influiscono sulle proprieta dell'atmosfera ed in particolare sulle grandezze di interesse operazionale per i veicoli e i propulsori.

Per derivare le equazioni che interpretano l'atmosfera quale campo fluidodinamico, trascureremo, fin dall'inizio, in una superficie di confine esterna arbitraria, verso lo spazio esterno, i trasferimenti di massa; riterremo cioe il campo a massa totale costante.

Isa seconda condizione al contorno, relativa alla energia entrante, e invece tenuta in conto; sara indicato con $\Phi$ il fiusso di energia (energia per unita di superficie e di tempo).

E noto che l'energia proveniente dal sole, sotto forma di radiazioni, viene in parte riflessa nello spazio (la frazione di energia totale che non raggiunge la Terra e $39 \%$ ); una parte viene assorbita dall'atmosfera " degradata dallo spettro visibile e dell'ultravioletto nello spettro dell'infrarosso $\left({ }^{2}\right)$.

Oltre alla energia pura di tipo radiante, energia associata a masse ponderali elementari e ceduta alla atmosfera.

I'assorbimento o degradazione dell'energia radiante e l'assorbimento delle radiazioni corpuscolari, si manifesta nelle equazioni del bilancio di energia, con la presenza di sorgenti di calore; indicheremo con 
$i_{1}$ e $\dot{q}_{2}$ la produzione di energia (sotto forma di calore) associata rispettivamente alle radiazioni pure e corpuscolari.

Si ammette, nella nostra analisi, che il fenomeno di assorbimento delle radiazioni corpuscolari avvenga per collisioni ionizzanti e che, nella mecanica di assorbimento e degradazione delle radiazioni pure, siano liberati elettroni (effetto fotoelettrico e di Compton).

Il fluido del campo reale, deve essere considerato, almeno localmente per certe fasce, elettricamente conduttivo, costituito da " $\mathrm{n}$ " componenti, reagenti chimicamente e sottoposto alle azioni di campi esterni, gravitazionali e magnetici.

Per derivare le equazioni, sono fatte le seguenti assunzioni:

i) il campo e ritenuto sistema "continuo " in tutta la sua estensione: questa ipotesi rappresenta il punto debole della base di calcolo dei modelli di atmosfera.

ii) è supposta valida la condizione di equilibrio termodinamico, ed è accettato il postulato fondamentale della termodinamica dei processi irreversibili, (si ammette cioe, che lo stato, nell'intorno di un punto, sia completamente determinato da un numero piccolo di grandezzo estensive). Questa seconda ipotesi non e una limitazione eccessiva, essendo "non accettabile " che eccezionalmente, per esempio, per zone che temporaneamente sono sede di una tempesta magnetica.

\section{2, III. - Le incognite del campo.}

In ogni campo fluidodinamico, si distinguono due tipi di incognite:

a) incognite fondamentali

b) incognite non fondamentali o derivate.

Le variabili indipendenti, nel caso pin generale sono le tre coordinate spaziali e la variabile tempo. Nello schema semplificato di atmosfera, adottato attualmente, sara tenuto conto solo di una delle quattro variabili indipendenti, la quota, ma è accertata l'influenza sulle proprieta dell'atmosfera delle variabili ignorate, in modo particolare della latitudine e del tempo. Le condizioni fisiche dell'atmosfera infatti variano con la latitudine, per effetto dello schiacciamento della terra ai poli e della variazione del campo magnetico terrestre; le variazioni col tempo, hanno curattere di continuita e, per un determinato fenomeno, e definibile un periodo caratteristico.

a) Le incognite fondamentali del campo (funzioni, nel caso piu generale delle tre coordinate spaziali e del tempo) sono: 
1) - Le incognite termodinamiche. Lo "stato" termodinanirco del sistema e caratterizzato da un numero di grandezze pari ai gradi di liberta; se « $n$ " e il numero di specie chimiche, individuano lo stato: $(n-1)$ densita parziali e due altre grandezze, per es. pressione $p$ e temperatura $T$; in totale $(n+1)$ grandezze termodinamiclie indipendenti.

La densita del costituente $\mathrm{n}^{\mathrm{mo}} \dot{\mathrm{e}}$ determinata con la relazione $\varrho=\sum_{1}^{n} \varrho_{i}$ o dalla relazione diflerenziale equivalente $\sum_{1}^{n}\left[d \varrho_{i}\right]=0$; per definizione $\varrho_{i o}=\frac{\rho_{i}}{\varrho}=\frac{\rho_{i}}{\sum_{1}^{n} \varrho_{i}}$.

Si noti:

- la equazione di stato dei gas perfetti vale in termini parziali: $p_{\imath}=\varrho_{i} \frac{R}{M} T$

- la equazione di stato in termini totali: $p=0 \frac{R}{M} T$ e derivata per sommatoria dalle equazioni in termini parziali $(*)$;

- le grandezze $M_{i}$ (pesi molecolari dei costituenti) sono noti, essendo note le specie chimiche presenti.

Le incognite fondamentali relative allo stato termodinamico sono:

$$
p, \quad T, \quad \varrho_{1}, \quad \varrho_{2} \quad \ldots \ldots \ldots \ldots \ldots, o_{(n-1)}
$$

oppure

$$
p, \quad T, \quad \varrho_{10}, \varrho_{2 o} \quad \ldots \ldots \ldots \ldots \ldots \varrho_{(n-1), 0}
$$

in totale $(n+1)$ incognite indipendenti.

La inclusione esplicita nelle equazioni della incognita addizionale "densita totale ", (che non è una incognita fondamentale), comportera la considerazione della equazione di stato (in termini totali).

2) una incognita cinematica " $v$ ", velocita media nel moto macroscopico di massa; grandezza vettoriale a tre componenti;

$\left(^{*}\right) \sum_{1}^{n} n_{i}-R T \sum_{1}^{n}\left(\frac{e_{i}}{M_{i}}\right) T$, proprieta estensiva, non e interessata dal segno di sonımatoria: $\sum_{1}^{n} p_{i}=p$ (legge di Dalton); $\sum_{1}^{n}\left(\frac{o_{i}}{I_{i}}\right)=0 \sum_{1}^{n}\left(\frac{n}{\varrho} \cdot \frac{1}{J \tilde{I}_{i}}\right)=$ $=O \sum_{1}^{n}\left(\frac{Q_{i o}}{I_{l}}\right)-\varrho \frac{1}{I I}$ essendo stato posto: $\frac{1}{H I}=\sum_{1}^{n}\left(\frac{\hat{s} i_{v}}{M_{l}}\right)$. 
3) le incognite elettriche; le seguenti: " $\varrho_{e}$ ", densita delle cariche elettriche: (grandezza scalare); " $E$ ", intensita del campo elettrico (grandezza vettoriale a tre componenti);

4) " $B$ ", intensita del flusso di induzione magnetica (grandezza vettoriale a tre componenti). In totale, sette incognite elettromagnetiche scalari.

Le incognite fondamentali del campo sono, in totale $(n+11)$.

b) Le incognite derivate.

Sono definite "derivate" grandezze del campo non fondamentali, ma che caratterizzano ulteriormente le proprieta termodinamiche, fluidodinamiche ed elettromagnetiche del campo. Nelle equazioni delcampo fluidodinamico in esame, saranno presenti alcune incognite derivate; esse saranno legate alle incognite fondamentali dalle cosidette equazioni costitutive.

3, III. - Il sistema di equazioni.

Sia " $P$ ", nel caso piu generale, una " proprieta " del campo fluido. Si possono verificare in un volume di controllo:

1) variazioni, nel tempo, della proprieta;

2) variazioni dipendenti dal moto medio macroscopico;

3) variazioni dipendenti dalla velocita microscopica o peculiare;

4) produzione della proprieta.

Le equazioni del campo, esprimenti bilancio di " proprieta ", nel caso piu generale, avranno un termine con la derivata temporale, un termine relativo agli effetti del moto macroscopico di massa, un termine relativo ai moti molecolari, un termine indicante produzione.

Riportiamo dalla bibliografia $\left({ }^{10}, 11,12,43,14\right)$, le equazioni del campo fluido, in esame; (i simboli sono specificati nelle pagg. 4 e 5).

$$
\frac{\partial}{\partial t} \varrho+\frac{\partial}{\partial r} \times(\varrho v)=0
$$

della continuita; scalare, una equazione

$$
\frac{\partial}{\partial t} \varrho_{i}+v \times \frac{\partial}{\partial r} \varrho_{i}+\frac{\partial}{\partial r} J_{i}=\varrho_{i}
$$

della diffusione; $(n-1)$ equazioni scalari 


$$
\begin{gathered}
\frac{\partial}{\partial t}(\varrho v)+\frac{\partial}{\partial r}(\varrho v \times v)+\frac{\partial}{\partial r} \times \mu^{+}=\varrho_{e} \omega^{\prime}+i \Lambda H^{\prime}+\mathrm{F}_{\text {est }} \\
\text { dell'equilibrio; vettoriale } 3 \text { equazioni }
\end{gathered}
$$

$$
\begin{aligned}
&\left.\frac{\partial}{\partial t} \mid \varrho\left(e+\frac{1}{2} v^{s}\right)\right]+ \frac{\partial}{\partial r} \times \varrho v\left(e+\frac{1}{2} v^{2}\right)+\frac{\lambda}{\partial r}\left(v P^{+}+J_{q}\right)=i \times E+\mathrm{F}_{\text {est }} \times \\
& \times v+q_{2}+q_{2} \\
& \text { dell'energia, scalare; una equazione }\left(^{*}\right)
\end{aligned}
$$

$$
\frac{\partial}{\partial r} \times D=o_{e}
$$

di conservazione delle cariche elettriche; scalare, una equazione

$$
\frac{\partial}{\partial r} \Lambda E-\frac{\partial B}{\partial t}
$$

I di Maxwell, vettoriale; tre equazioni scalari

$$
\frac{\partial}{\partial r} \Lambda H=i+\frac{\partial D}{\partial t}
$$

II, di Maxwell, vettoriale, tre equazioni scalari.

In totale si dispone di $n+11$ equazioni.

È da notare:

1) La presenza della incognita addizionale "densita " (oo), non fondamentale, richiede l'uso della equazione di stato, che da questo punto in avanti riterremo costantemente una equazione del sistema.

2) Nel gruppo di equazioni fluidodinamiche compaiono i seguenti flussi microscopici, definiti in funzione delle velocita peculiari; $\left({ }^{15}\right)$

\section{$J_{i}$}

flusso microscopico (dovuto alle velocita peculiari) di massa

(*) L'equazione dell'energia utilizzata nello studio dei campi fluidodinamici con reazioni chimiche assume l'aspetto equivalente seguente: (dopo avere espresso, dovunque sia possibile, le grandezze in funzione dei calori di formazione dei costituenti e tenendo presente nella equazione della energia, della equazione della diffusione, ed esplicitando il flusso di calore, $\left.{ }^{(15}\right)$ :

$$
\begin{aligned}
\varrho \frac{D}{D t}\left(c_{p} T\right. & \left.+\frac{1}{2} v^{2}\right)-\frac{\partial}{\partial !} p+\frac{\partial}{\partial r} \times(v \times \tau)+\frac{\partial}{\partial r} \times\left[-\lambda \frac{\partial T}{\partial r}+\Sigma c_{p} T J_{i}\right]+ \\
& +\Sigma_{i} h_{i} \dot{\varrho}_{i}=i \times \mathrm{E}+\mathrm{F}_{\mathrm{est}} \times v+\dot{q}_{i}+\dot{q}_{2} .
\end{aligned}
$$

Nella nostra analisi preferiamo adottare la forma non elaborata della equazione dell'energia, che mostra in modo piu evidente il bilancio energetico. 


$$
P^{+}=P_{1}+P_{2}+P_{3}
$$

tensore degli sforzi; contiene in contributo $P_{3}$ degli efietti dissipativi di natura elettromagnetica; $P_{1}=p C$ e la parte reversibile del tensore degli sforzi; $P_{2}$ e il tensore degli sforzi dissipativi viscosi

$J_{q}$

flusso di energia sotto forma di calore.

Per la soluzione del problema matematico, è necessario collegare le grandezze microscopiche alle macroscopiche, cioe esplicitare i flussi in funzione di grandezza macroscopiche. La mecanica statistica stabilisce le relazioni necessarie $\left({ }^{15}\right)$, in funzione di coefficienti fenomenologici, rome per es.: $D_{i j}$ coefficiente di diffusione: $\mu$ coefficiente di viscosita; $\lambda$ coefficiente di conducibilita termica.

3) Il termine produzione $\varrho_{1}$, che ha il significato di rapidita, rispetto ad un "tempo chimico", di produzione del costituente; simbolicamente: $\dot{Q}_{i}=\frac{\partial}{\partial t_{c h}} \underline{O}$.

Le espressioni di $\underline{g}_{i}$ sono fornite, con approssimazione non molto soddisfacente (cio è messo in rilievo (11)) dalla cinetica chimica.

1) Nel gruppo di equazioni elettromagnetiche compaiono le seguenti incognite addizionali: " $H$ ", vettore forza magnetica " $D$ », flusso di induzione elettrostatica; " $i$ ", vettore, densita della corrente di induzione. La tripla indeterminatezza, rappresentata dalle tre incognite addizionali, e sciolta da tre equazioni; due (costitutive) legano le grandezze derivate a incognite fondamentali:

$$
\begin{aligned}
& D=f_{1}(B, E, \text { stato }) \\
& H=f_{2}(B, E, \text { stato })
\end{aligned}
$$

la terza e la equazione dinamica di Ohm:

$$
i=f_{3}(B, E, \text { stato }) .
$$

Le tre relazioni, in casi particolari (14), hanno l'aspetto semplice:

$$
\begin{aligned}
D & =\varepsilon E \\
B & =\mu+H \\
i & =\varrho_{e} v+\sigma[E+v \Lambda B]
\end{aligned}
$$

essendo $\varepsilon, \mu, \sigma$ costanti del mezzo.

i da notare che i due gruppi di equazioni (fluidodinamiche ed elettromagnetiche) non sono "separabili », cioe il sistema di $(n+11)$ equa- 
zioni del campo deve essere risolto contemporaneamente. La interdipendenza dei due gruppi di equazioni appare dalle equazioni della fluidodinamica dell'equilibrio e della energia nelle quali sono contenuti termini con forze elettrodinamiche e dalla equazione dinamica di Ohm, che contiene il termine velocita media macroscopica di massa.

\section{4, III. - Il sistema semplificato di equazioni.}

Indipendentemente dalla difficolta di ottenere la soluzione, in forma chiusa, del sistema generale di equazioni del campo, ha interesse, per la pratica compilazione di tabelle della atmosfera, un sistema semplice di equazioni. La semplicita e integrabilita del sistema si raggiungono attraverso una serie di ipotesi che rappresentano il graduale abbandono delle basi generali di calcolo.

Introduciamo progressivamente le ipotesi restrittive, puntualizzando le conseguenze sia sulla base fisica di calcolo che sul bilancio tra equazioni disponibili ed incognite del campo

a) $\varrho_{e}-E=B=0 \quad$ (e di conseguenza $i=D=H=O$ ).

La posizione di nullita di tutte le grandezze di carattere elettromagnetico comporta l'assenza, nell'atmosfera, di fascie ionizzate, di cariche libere e di effetti incrociati elettromagnetici. (In un mezzo di queste caratteristiche le comunicazioni radio sarebbero impossibili).

Praticamente si assume la inesistenza di collisioni ionizzanti delle particelle ponderali, (le quali, peraltro non influenzano il bilancio di massa), né si ammette la degradazione delle radiazioni, che sarebbe accompagnata da emissione di elettroni (effetto fotoelettrico, effetto Compton).

Naturalmente non si ammette accumulo di energia nella atmosfera; bisogna ritenere idealmente che la seconda superfice di confine, materialmente rappresentata dalla superfice terrestre, sia trasparente ai flussi energetici che la colpiscono.

Il flusso di energia, di qualunque natura non provoca effetti, cioe l'atmosfera e indifferente al flusso che l'attraversa; nel bilancio, tale situazione coincide con $\frac{\partial}{\partial r} \Phi=0$, ovvero con la posizione equivalente $\dot{q}_{1}=\dot{q}_{2}=0$.

Le equazioni elettromagnetiche del sistema vengono perdute, e le equazioni fluidodinamiche di continuita e dell'energia acquistano un aspetto piu semplice. Il tensore degli sforzi $P^{+}$perde il contributo dissipativo di natura elettromagnetica. Il sistema piu semplice, ottenuto 
dal sistema generale, rimane determinato: $(n+4)$ equazioni disponibili e $(n+4)$ incognite:

$$
J_{1}=P_{2}=J_{q}=0 \text {. }
$$

La posizione equivale ad assumere la trascurabilita degli effetti dissipativi del moto molecolare (fluido non viscoso, non conduttivo ecc.). Il sistema diventa ulteriormente piu semplice mentre non viene alterato ne il numero di equazioni ne il numero di incognite.

La posizione piu restrittiva e $J_{q}=0$ (assenza di flussi di calore); che falsifica, rispetto alla realta, ancora ulteriormente il bilancio energetico nell'atmosfera superiore, dopo le posizioni $q_{1}=q_{2}=0$ :

$$
\dot{Q}_{i}=0 \text {. }
$$

La posizione di nullita della " produzione" di massa per gli $n$ componenti, interpreta la condizione di equilibrio chimico; le concentrazioni sono corrispondenti alle condizioni di equilibrio (che puo subire spostamenti), i singoli componenti hanno distribuzione maxwelliana della velocita. La posizione lascia inalterato il bilancio tra equazioni e incognite: $(n+4)$ equazioni; $(n+4)$ incognite:

$$
v=0 \text {. }
$$

Il campo è ritenuto statico; come conseguenza delle posizioni $\alpha, \beta$, $\gamma, \delta$, esso risulta anche stazionario; ( $v$ e la velocita relativa alla terra).

Rimane disponibile una unica equazione; (le altre equazioni, diventano identita); quella di equilibrio di espressione semplicissima:

$$
\frac{\partial}{\partial r} \times p U=\mathbf{F}_{\mathrm{est}} \text {, }
$$

alla quale, per quanto è stato detto nel paragrafo precedente (3, III), si associa la equazione di stato dei gas pertetti.

Il sistema di equazione risultante è dunque il seguente:

$$
\left\{\begin{array}{r}
\frac{\partial}{\partial r} \times p U=\mathrm{F}_{\mathrm{est}} \\
p=\varrho \frac{R}{M} T
\end{array}\right.
$$

$M=\left[\sum_{1}^{n}\left(\frac{\varrho_{i o}}{M_{i}}\right)\right]^{-1}$, peso molecolare totale; dipende da spostamenti dell'equilibrio chimico, (la condizione è espressa da $\dot{o}_{\mathfrak{\imath}}=0$, come si è visto). La soluzione delle equazioni $\dot{o}_{i}=0$, dipendenti da una cinetica 
chimica complicata, non sara ricercata; si fa assumere a $M$ invece il ruolo di variabile essenziale del campo.

Le incognite del campo risultato: $p$ (pressione); $\varrho$ (densita), $T$ (temperatura), $M=\left[\sum_{1}^{n}\left(\frac{\varrho_{i o}}{M_{i}}\right)\right]^{-1}$, peso molecolare totale.

$\varepsilon)$

$$
r=Z
$$

La ulteriore ed ultima posizione $\varepsilon$, secondo cui la unica valiabile spaziale di interesse e l'altitudine, conferisce al sistema di equazioni, l'aspetto noto:

$$
\left\{\begin{array}{l}
\frac{d p}{d Z}=-\varrho g \\
p=\varrho \frac{R^{+}}{M} T .
\end{array}\right.
$$

La prima relazione e la equazione dell'equilibrio idrostatico; " $g$ " e il valore del campo gravitazionale terrestre, noto in funzione della quota, attraverso la relazione di Lambert ${ }^{\left({ }^{16}\right)}$. Il campo gravitazionale della Luna, i cui effetti sono vistosi sulla Terra, con le maree, e stato trascurato.

Un'atmosfera, rappresentata dal sistema semplificato di equazioni, non presenta dissipazioni, cioe sorgenti di entropia, e un sistema immobile, a massa ed energia costanti, ed è indifferente ai flussi provenienti dall'esterno (ad un flusso entrante, rispetto ad una superficie esterna convenzionalmente fissata, corrisponde un flusso uscente, attraverso una superficie di confine interna).

Appare chiaro, in definitiva, che da un sistema complicato di equazioni, di validita generale, e determinato, attraverso successive posizioni, si e giunti ad un sistema di validita ristretta, semplice, ma due volte indeterminato. L'effetto più vistoso e negativo del processo di semplificazione matematica e la necessità di sopperire alla doppia indeterminatezza, con sondaggi sperimentali.

IV. - CALCOLO NUMERICO DELL'A TMMOSFERA.

Riportiano gli elementi fondamentali del calcolo numerico della atmosfera, facendo riferimento al modello dell'Air Force, ARDC 1959. 
Dalla combinazione delle due equazioni del campo, si ha:

$$
\frac{d p}{p}=-\frac{g M}{R^{+} T} d Z
$$

Naturalmente il grado di indeterminatezza si trasferisce inalterato dalle equazioni di partenza, alla combinazione di esse.

Per poter giungere ad espressioni utili per la tabulazione si introducono relazioni:

a)

$$
\begin{gathered}
g(Z) d Z=G d H^{\prime} \\
T_{M}=T \frac{M_{0}}{M}
\end{gathered}
$$

" $G$ ", nella prima relazione è una costante dimensionale;

" $H^{\prime}$ ", e un nuovo parametro indipendente (che sostituisce $Z$ ); la relazione di Lambert $g=f(Z){ }^{(16)}$ e determinata per via autonoma.

" $H^{\prime}$ " e definito " geopotenziale " ed e il lavoro richiesto per portare la unita di massa, a partire da una posizione standard del campo gravitazionale terrestre alla quale, convenzionalmente, si ritiene nullo il valore dell'energia potenziale del campo, (livello del mare), fino alla quota geometrica $Z$.

L'unita adottata per " $H^{\prime}$ " e il metro geopotenziale (m con apice: $\left.\mathrm{m}^{\prime}\right)$; il valore di $G$, con questa scelta, e: $G=9,80665 \mathrm{~m}^{2} \mathrm{sec}^{-2} \mathrm{~m}^{\prime-1}$.

Alla quota geometrica $Z$ corrisponde una quota geopotenziale $H$, espressa, nella unita scelta, da un valore minore (in conseguenza della diminuzione del campo gravitazionale con la quota).

La seconda combinazione di variabili definisce " $T_{M}$ "; temperatura in scala molecolare (" molecular scale temperature "); per definizione. $T_{M} \dot{\boldsymbol{\theta}}$ funzione della temperatura e della composizione della atmosfera.

L'equazione differenziale, dopo avere sostituito in essa le relazioni [1] e [2], diventa:

$$
\frac{d \ln p}{d H^{\prime}}=\left(\frac{G M_{0}}{R^{+}}\right) \frac{1}{T_{M}},
$$

il termine in parentesi è un numero dimensionale costante

$$
\left(-\frac{G M_{o}}{R^{+}}=Q=0,0341648 \frac{o k}{w^{\prime}}\right) \text {. }
$$

$\left(^{*}\right)$ Il valore reciproco negativo del coefficiente di $d Z: \psi=\frac{R+T}{g \pi}$ ha le dimensioni di una lunghezza ed e chiamato "scala delle quote" (scale height nella terminologia anglosassone); rappresenta l'altezza di una atmosfera fittizia omogenea di densita uniforme, a temperatura costante $T:\left({ }^{17}\right)$. 
Il sostanziale progresso, nel passaggio dalla $(1, \mathrm{IV})$ alla $(3, \mathrm{IV})$, consiste nella conservazione della semplicita di partenza ed il parallelo superamento della necessita di introdurre, per la integrazione, le posizioni non valide dappertutto $g=$ cost; $M=$ cost.

La determinazione teorica o sperimentale della funzione $T_{M}=f\left(H^{\prime}\right)$ cioe la conoscenza delle leggi di variabilita della temperatura e delle concentrazioni, in funzione della quota, sciogliendo la doppia indeterminatezza del sistema di equazioni del campo, permette la tabulazione cercata.

Difficolta tecniche pero impediscono di fare misure dirette, attendibili, in quota, della temperarura e delle concentrazioni; del resto il campo di azione dei raggi sonda vettori della strumentazione di misura, e modesto (intorno a $200 \mathrm{~km}$ ).

La grandezza fisica misurabile piu facilmente e la densita; inoltre dati relativi alla densita sono deducibili teoricamente con il calcolo, attraverso la osservazione degli effetti di resistenza dell'atmosfera sui satelliti artificiali in orbite terrestri.

Sono dunque utilizzate, per sciogliere il doppio grado di indeterminatezza del campo, determinazioni di densita. I passi del procedimento di calcolo sono i seguenti:

1) Riadattamento della equazione $(3, \mathrm{IV})$ per far comparire in essa la variabile "densita "; si ha: $\left({ }^{16}\right)$

$$
\frac{d}{d H^{\prime}} \ln \varrho=\frac{1}{T_{M}}\left(Q+\frac{d T_{M}}{d H^{\prime}}\right)
$$

(" $Q$ " e la combinazione dimensionale di costanti prima introdotta).

2) Determinazione con sondaggi o per via teorica della funzione $\varrho=f\left(H^{\prime}\right)$.

3) Determinazione, per tentativi del gradiente $\frac{d T_{u}}{d H^{\prime}}$, a partire dai dati di densita disponibili.

4) Determinazione delle concentrazioni con la quota, al fine di determinare la $M-f(H)$. La difficolta di avere misure dirette attendibili, di cui si e gia riferito di $M=\left[\sum_{1}^{n}\left(\frac{\varrho_{10}}{M_{i}}\right)\right]^{-1}$ e la necessita di ricorrere a speculazioni teoriche $\left({ }^{16,}{ }^{17}\right)$ rappresentano un altro punto debole dell'attuale metodo di calcolo. La conoscenza della legge di variazione $M-f(H)$, permette di ricavare la variabilita, con la quota, della temperatura. 
Si rileva che il numero delle determinazioni autonome, sperimentali e teoriche, non e alterato dai successivi riadattamenti della equazione del campo; per il modello di atmosfera $\triangle R D C$ 1959, le misure dirette riguardano la densita e il peso molecolare (che, come era stato previsto, aquista il valore di variabile essenziale).

\section{V. - DETERMINAZIONI SPERTMENTALI.}

\section{1, V. - Determinazioni di densita.}

Le misure di densita sono fatte direttamente con razzi, con tecniche di misura diversissime (18, 19, 20, 21, 22); (osservazione della espansione di una nube di sodio, con equipaggiamenti miniaturizzati, ecc.).

Raccogliendo nella seguente tabella, i dati piu importanti relativi ad alcuni razzi di ricerca $(18,18,20,21,22)$ :

\begin{tabular}{|l|c|c|}
\hline \multicolumn{1}{|c|}{ Nome } & $\begin{array}{c}\text { Quota operativa } \\
\text { (Miglia) }\end{array}$ & $\begin{array}{c}\text { Carico strumentale } \\
\text { (lb) }\end{array}$ \\
\hline Aerobe & 150 & 150 \\
Mike-Cayum & 100 & 50 \\
Spaerobee & 350 & 40 \\
Terrapin & 80 & 6,5 \\
\hline
\end{tabular}

In generale, le misure di densita con razzi sono possibili fino a $200 \mathrm{~km}\left(^{\ominus}\right)$, i lanci a quota maggiore diventano notevolmente piu costosi e dunque proibitivi. Per il modello dell'atmosfera dell' $A$ ir Force in esame, (ARDC 1959) i dati sono numerosi fino a poco meno di $200 \mathrm{~km}$, solo qualche dato è disponibile per quote intorno a $350 \mathrm{~km}$. La localita di lancio sono White Sands e Fort Churchill ( $\left.{ }^{16}\right)$.

A quote piu alte, la densita dell'aria si rileva, attraverso le osservazioni della variazione, causata dal drag aerodinamico dell'orbita dei satelliti artificiali.

A causa della presenza di atmosfera [la quale si estende poco in quota, dal punto di vista della sostentazione aerodinamica, e si estende notevolissimamente dal punto di vista della resistenza; $\left.\left({ }^{23}\right)\right]$, non e possibile far percorrere ad un satellite artificiale un'orbita ad energia costante. Per effetto della dissipazione di energia il satellite si spostera continua- 
mente su nuove ellissi, ognuna delle quali e ellisse oculatrice della traiettoria effettiva; di conseguenza gli elementi geometrici della ellisse sono variabili.

La integrazione della equazione del moto (centrale) del satellite, fornira una relazione tra causa (densita dell'aria) ed effetto (variazione dei parametri geometrici dolla ellisse, rispetto al caso teorico di assenza di resistenza); cioe saranno disponibili relazioni del tipo:

$$
\begin{aligned}
& a=f_{1}\left(\varrho_{1} C_{D}\right) \\
& e=f_{2}\left(\begin{array}{ll}
\varrho_{1} & C_{D}
\end{array}\right)
\end{aligned}
$$

" $a$ " semiasse maggiore della ellisse;

" $e$ " eccentricita $(*)$;

" $\varrho$ " densita (caratteristica del mezzo);

$C_{D}$, coefficiente di resistenza (caratteristica aerodinamica del satellite), determinata sperimentalmente $(* *)$.

Il rilevamento, da terra, della traiettoria del satellite e la conoscenza di $C_{D}$, preliminarmente acquisita in laboratorio, permette il calcolo di " $\varrho$ ".

Il valore di $C_{D}$ dipende dalla orientazione del corpo; per il cilindro del satellite Explorer e stata trovata, per il $C_{D}$, una variazione, con l'orientamento dell'asse geometrico, nel rapporto 1 a $10\left({ }^{16}\right)$.

Rinviamo il lettore alla bibl. $\left({ }^{24}, 25,26\right)$ per l'esame dettagliato della misura della densita con i satelliti; diamo alcuni dati geometrici (apogeo

\begin{tabular}{|c|c|c|c|}
\hline Nome Satellite & $\begin{array}{l}\text { Perigeo } \\
\text { (miglia) }\end{array}$ & $\begin{array}{c}\text { Apogeo } \\
\text { (miglia) }\end{array}$ & Tipo di missione \\
\hline Discoverer XXIII & 126 & 882 & $\begin{array}{l}\text { Osservazioni degli efletti di re- } \\
\text { sistenza (ovvero determinazione } \\
\text { della densita) }\end{array}$ \\
\hline Discoverer XXIV & 139,1 & 251 & 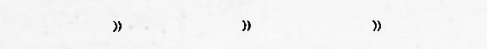 \\
\hline Discoverer $\mathrm{XXV}$ & 146 & 503 & $”$ \\
\hline
\end{tabular}
e perogeo) di alcuni satelliti da ricerea (tutti lanciati nel 1961) $\left({ }^{27}\right)$.

$\left({ }^{*}\right)$ Nei calcoli di Sterne $\left({ }^{24}\right)$, utilizzati nel modello di atmosfera in questione, i parametri, geometrici adottati sono: $r_{a}$, distanza del satellite all'apogeo; $r_{p}$, distanza al perigeo. Risulta:

$$
\left(\frac{r_{p}+r_{a}}{2}\right)=a ;\left(\frac{r_{a}-r_{p}}{r_{a}+r_{p}}\right)=e \text {. }
$$

$\left(^{* *}\right)$ La determinazione sperimentale di $C_{D}$ richiede tecniche sperimentali appropriate, dovendo essere simulato un ambiente estremamente rarefatto. 
I dati disponibili per il modello "ARDC 1959 ", sono stati dati da satelliti operanti tra 170 e $650 \mathrm{~km}$ di quota.

I valori numerici del modello, sopra la quota di $700 \mathrm{~km}$, rappresentano dunque un tentativo di estrapopolazione ${ }^{\left({ }^{16}\right)}$.

\section{2, V. - Determinazione di peso molecolare.}

Nel modello in esame, l'atmosfera è ritenuta secca, di composizione costante fino a $90 \mathrm{~km}^{\prime}$ (geopotenziali) ${ }^{\left({ }^{16}\right)}$ costituita da $n=11$ componenti [vedi tabella in $\left({ }^{16}\right)$ ] .

Il peso molecolare medio risultante, al livello del mare, $M_{0}$, hat valore 28,966 e rimane costante fino a $90 \mathrm{~km}$. Alle quote superiori, per effetto delle variazioni di composizione, è invece, variabile.

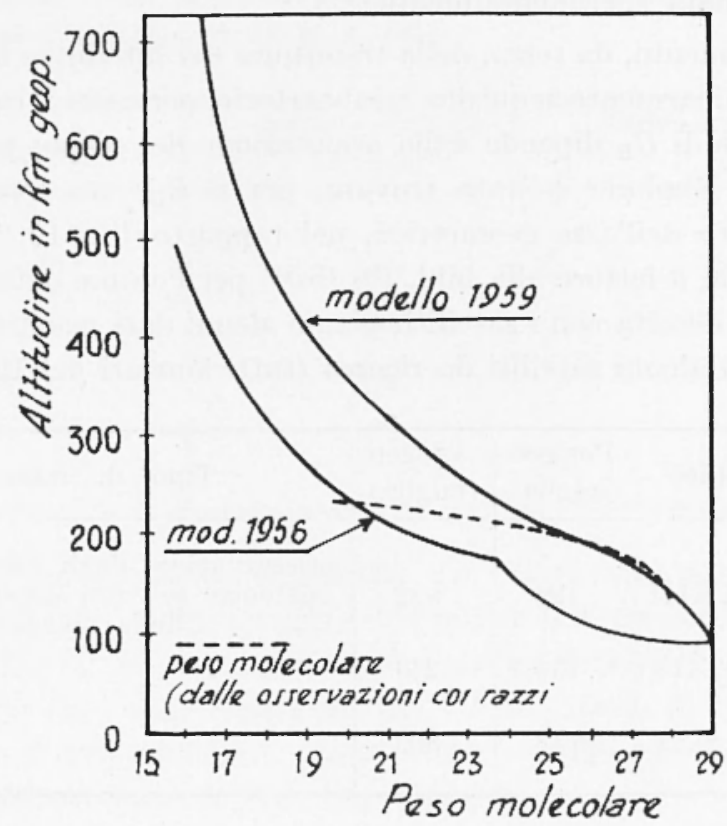

La curva del peso molecolare in funzione della quota geopotenziale, per il modello ARDC 1959, rappresenta un tentativo di utilizzazione dei dati sperimentali disponibili $\left({ }^{16}\right)$ riportati da Townsend, raccolti con spettometri di massa del Bennett, portati in quota da razzi $\left({ }^{16}\right)$.

Il diagramma [riportato $\left({ }^{16}\right)$ ] indica:

1) la curva relativa al modello precedente, ARDC 1956, ottenuta per via puramente teorica, e basata sulla assunzione che responsabile 
della variazione di peso molecolare era unicamente l'ossigeno, la cui dissociazione inizia a $90 \mathrm{~km}^{\prime}$ ed e completa a $175 \mathrm{~km}^{\prime}\left({ }^{18}\right)$;

2) La curva dei dati sperimentali (tratteggiata), ottenuta portando in quota, con i razzi, la strumentazione di misura.

3) La curva del modello 1959, stabilita con la utilizzazione dei dati sperimentali e la imposizione della condizione di congruenza fisica rappresentata dalla relazione $\frac{d T}{d H^{\prime}}>0$ (conseguenza dei fenomeni di dissociazione $\left.\left({ }^{16}\right)\right)$.

Alla base dei calcoli teorici del modello ARDC 1956 era l'ipotesi della variazione delle concentrazioni, secondo l'equilibrio diffusivo $\left({ }^{*}\right)$, secondo la legge cioe: $\varrho=\varrho_{o} e^{-v Z}$ essendo $v=\frac{m g}{R^{+} T}$, con " $m$ " peso molecolare di un individuo della miscela costituente l'aria.

La notevole diversita quantitativa delle leggi di variazioni "peso molecolare-quota", relativo ai modelli ARDC 1956 e ARDC 1959, si riflette sui valori numerici delle grandezze tabulate $\left({ }^{16}\right)$, frequenza delle collisioni, cammino medio molecolare, temperatura cinetica, pressione ecc.)

Sono riportati appresso i valori numerici di alcune grandezze ricorrenti nei problemi missilistici relativi ai due modelli ARDC 1956 e ARDC 1959.

VI - ANalisi critica.

a) Critica alla base matematica.

1a) Indipendentemente dalle rinuncie sostanziali accettate per isolare, dal sistema iniziale complicato, il sistema semplice ma indeterminato, appare molto grave l'ammissione di validita in qualunque zona della atmosfera delle due equazioni, barometrica e di stato.

Cio equivale ad ammettere che il campo fluido rappresentato dall'atmosfera, possa essere descritto, in qualunque zona, dalle proprieta

(*) Dobbiamo segualare che l'edizione Italiana "ARDC 1956 " calcolata dal Centro nazionale di calcolo, a cura del Centro Studi Propulsione a Reazione ${ }^{12}$ ) contiene la notevolissima arbitrarieta, rispetto al corrispondente modello americano, di tenere costante il peso molecolare dell'atmosfera tra 0 e $500 \mathrm{~km}\left({ }^{16}\right.$, p. 2$)$; viene con questo ignorata la dissociazione in quota e gli effetti del sopraggiungere dell'equilibrio diffusivo.

macroscopiche che compaiono nelle due equazioni (pressione, tempera- 


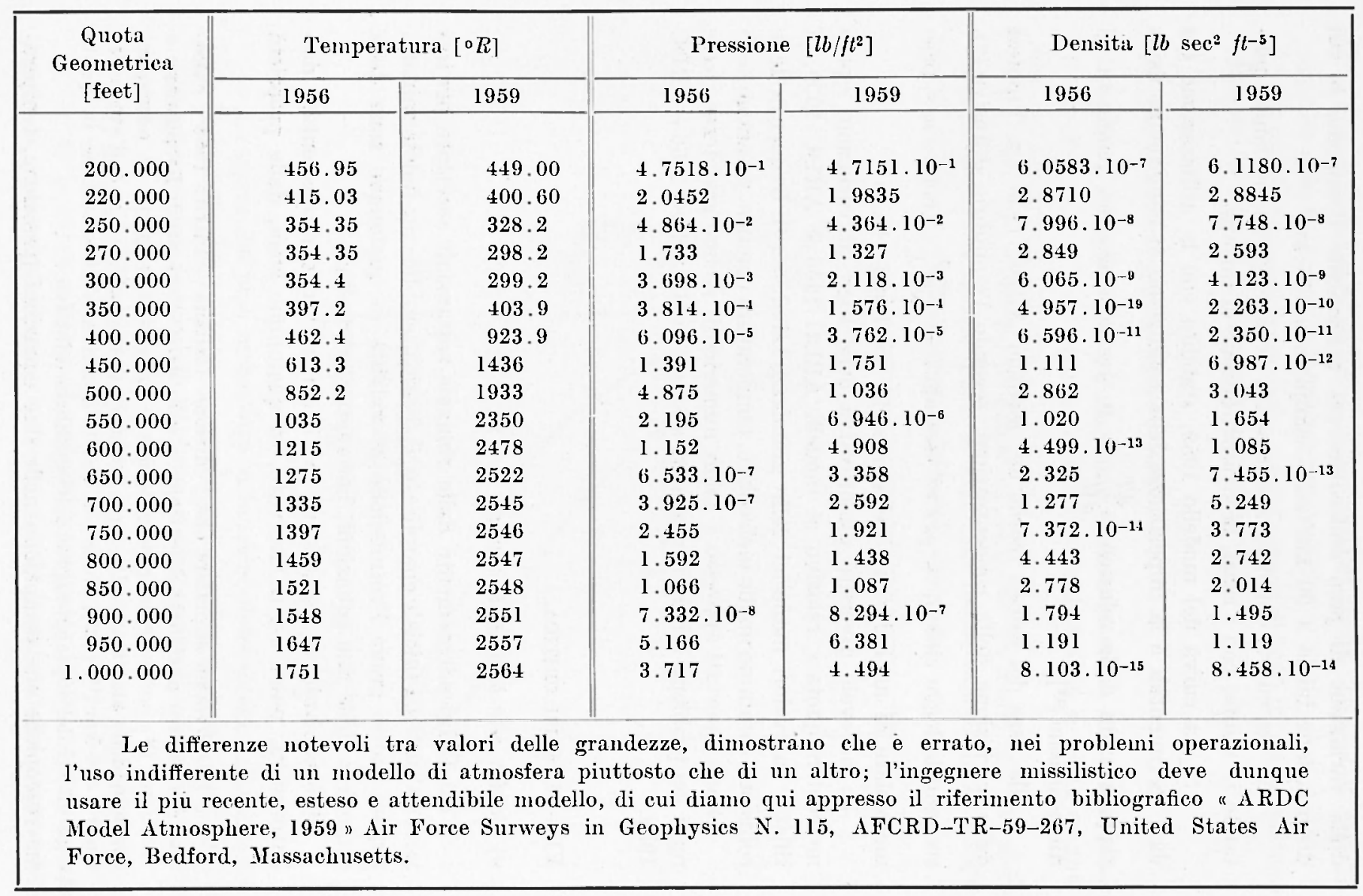


tura, densita e peso molecolare), cioe equivale ad accettare come verificato dappertutto il concetto di "continumm ».

La posizione fittizia di materia continua, molto produttiva in tutti i problemi usuali di fluidodinamica, non e piu aceettabile nell'alte zone dell'atmosfera, poiche in un volume di controllo, il piu piccolo di interesse, (praticamente il minimo capace di contenere un missile), il numero di molecole non puo essere ritenuto sempre infinitamente grande, ne le molecole agiscono in continua frequentissima collisione.

Qualunque problema, nelle zone in cui l'ipotesi del continuo non e rispettata, dovrebbe essere trattato, analizzando il comportamento individuale di ogni molecola o di gruppi di molecole, statisticamente.

In conclusione alle alte quote l'atmosfera, nel senso ordinario del termine, non esiste piu; la sua struttura e la sua fisica sono rivoluzionate ( la pretesa di continuare la sua descrizione in termini macroscopici rappresenta una liberta molto grande.

2a) Le equazioni differenziali del moto del satellite, non sono integrabili in forma chiusa. La loro integrazione si fa per quadratura $\left({ }^{24}\right)$, di conseguenza i risultati sono approssimati (sebbene l'errore possa essere contenuto teoricamente in limiti prestabiliti).

3a) Per il calcolo della velocita media della particella di aria " $V$ ", definita come media aritmetica della distribuzione maxwelliana delle velocita, si assume che l'atmosfera sia costituita da molecole di tipo unico (non miscuglio di gas diversi), aventi caratteristiche medie.

ta) Per il calcolo del coefficiente di viscosita si e rinumciato alla definizione di esso sul piano statistico della teoria cinetica (Chapmann) e si è assunta valida una relazione empirica (di Sutherland) nella quale la viscosita dipende solo dalla temperatura $\left({ }^{16}\right)$.

5a) Inoltre per la determinazione di " $\mathrm{L}$ " (cammino libero molecolare, cioe distanza probabile tra due collisioni successive) si e assunto per l'atmosfera il modello di gas omogeneo, le cui molecole sono di forma sferica, hanno una distribuzione maxwelliana delle velocita ed hanno urti molecolari completamente elastici.

6a) L'espressione della velocita del suono contiene implicitamente l'accettazione della validita, per l'atmosfera, delle equazioni di stato per' i gas perfetti.

b) Critica della base sperimentale.

1b) I dati raccolti dalla strumentazione di razzi si riferiscono in buona parte alla latitudine di Fort Churchill, nella base di Hudson che 
e vicino al polo magnetico. Questa particolare dislocazione del punto di raccolta dei dati, a causa dell'alta energia del campo magnetico terrestre in quella zona, influisce con una diminuzione del rapporto ossigeno atomico, (dissociato) a ossigeno molecolare, rispetto ad altri punti dell'atmosfera terrestre.

La discordanza del peso molecolare riscontrata all'altitudine di $200 \mathrm{~km}$ tra i valori per il modello 1956 ed il modello 1959 può considerarsi attenuata a latitudini minori $\left({ }^{16}\right)$.

2b) Esistono regioni dell'atmosfera (nel campo in cui sono forniti valori numerici delle proprieta) non sufficientemente esplorate.

Alla quota di $219 \mathrm{~km}^{\prime}$ e usato un unico valore della densita $\left({ }^{16}\right)$; per quote inferiori, e stato possibile considerare valori medi della densitas

3b) La dipendenza dei valori numerici della densita della geometria del corpo e dal coefficiente di resistenza è causa di errori.

La sezione trasversale effettiva del satellite, dalla quale dipende l'entita della resistenza aerodinamica, non e costante. Essa varia sulla traiettoria con l'orientamento disordinato e incontrollato dell'asse geometrico $\left({ }^{16}\right)$, di conseguenza i valori di densita calcolati con i satelliti, rappresentano indicazioni variabili entro un determinato campo. Nella figura di $\left({ }^{16}\right)$ (profilo della densita con la quota) viene indicato, per diverse zone, il campo di incertezza della determinazione di densita.

4b) Inoltre, il coefficiente di resistenza per corpi che si muovono in un mezzo rarefatto, è stabilito in laboratorio e la sua determinazione sara certamente affetta da errore sperimentale.

\section{VII - CONCLUSIONE.}

La conclusione che deriva dall'analisi e dalla critica sviluppata nella nota, e la seguente: i modelli di atmosfera compreso il più recente, debbono essere ritenuti non definitivi.

1) Le basi teoriche di calcolo, fin adesso adottate, sono valide in confini ristretti. L'uso indiscriminato delle equazioni risolutrici in tutte le zone dell'atmosfera, rappresenta una cosciente accettazione di approssimazione.

2) Le basi sperimentali, a loro volta, sono insufficienti e spesso informazioni isolate, provenienti da strumentazione non completamente collaudata, non sono verificate da successive misure. Nel futuro si pre- 
vede ed e auspicabile, che i modelli di atmosfera siano verificati con una raccolta sistematica ed estesa di dati.

3) L'attuale, piu attendibile modello dell'Air Force deve ritenersi modello approssimato, capace di fornire solo valori medi delle proprieta, per la particolare latitudine di Fort Churchill, il quale come e segnalato, e in prossimita al punto singolarissimo rappresentato dal polo magnetico.

4) La revisione dei modelli, è urgente, per la non validita della ipotesi della continuum, (secondo Singer, al di sopra di $500 \mathrm{~km}$ di altitudine) (*).

(*) Vogliamo indicare in questa nota, che e successiva alla compilazione della relazione e alla lettura al IX convegno, i piu recenti progressi relativi alle ricerche sulla atmosfera superiore.

Nell'Agosto 1962 e stato annunciato l'approntamento di un ulteriore modello di atmosfera, che sara divulgato con il titolo: "The IT.S. Standard Atmosphere - 1962 " come modello della COESA (U.S. Committee on Extension to the Standard Atmosphere) (Vedi "Astronautics" Agosto 1962); le tabelle rappresentano la continuazione degli sforzi di affinamento dei valori numerici delle proprieta dell'alta atmosfera.

Il lavoro del gruppo della COESA iniziava nel Gennaio 1960, con lo scopo di revisionare i dati disponibili, raccolti con i sondaggi o ottenuti con i satelliti.

Nello sviluppo degli studi, provvisoriamente, era raccomandata l'adozione del modello "ARDC 1959" (questa raccomandazione e in perfetto accordo con i risultati della nostra indagine). La nota relativa al " the U.S. Standard Atmosphere 1962 " comparsa sulla rivista "Astronautics" (della "American Rochet Society ", numero di Agosto 1962) mette in rilievo che i dati utilizzati per il modello ARDC 1959 erano stati raceolti durante il periodo di massima attivita solare dunque il modello doveva essere ritenuto come rappresentativo delle condizioni eccezionali corrispondenti, piuttosto che di condizioni medie normali.

Il modello revisionato "U.S. Standard Atmosphere 1962 " sara basato invece sui valori medi di determinazioni ottenute in corrispondenza di attivita solare con il massimo e il minimo di macchie solari.

Nella nota di "Astronauties" e riportato un elenco di organizzazioni partecipanti al programma della COESA, e viene dato lo schema del nuovo modello di atmosfera (che si estende fino a $700 \mathrm{~km}$ ) e che presenta notevole diversita dei valori numerici rispetto agli altri precedenti, compreso il modello ARDC 1959.

Nei programmi della COESA e anche l'approntamento di tabelle dell'atmosfera, fino all'altitudine di $90 \mathrm{~km}$ relativa a valori medi di proprieta dell'atmosfera in estate $e$ in inverno, con un intervallo di 25 gradi, tra i tropici e i poli.

Il modello di atmosfera della COESA appena disponibile, sara il piu attendibile e il pii recente e dovra essere adottato dall'ingegnere missilistico, in sostituzione del modello ARDC 1959. 


\section{BIBLIOGRAFIA}

(1) Ministero della Difera Aeronantica, Manuale dell'Atmosfera tipo ICAO. Roma, 1954.

$\left({ }^{2}\right)$ International Civil Aviation Organization, Manual of the ICAO Standard Atmosphere. Montreal, Canada (May 1954).

$\left({ }^{3}\right)$ Mlanual of the ICAO Standard Atmosphere Calculation by the NACA, Naca T.N. 3182, Washington (May 1954).

(4) Standard Atmosphere Tables and Data for Altitude up to $65800 \mathrm{fts}$. Naca Rep. No. 1235, (1955).

$\left({ }^{5}\right)$ Minzner R. A., Ripley, W. S. and Condron T. P., U.S. Extension to the $I C A O$ Standard Atmosphere. U.S., Government Printing Office, Washington, D.C. (1958).

$\left({ }^{6}\right)$ Minzwer R. A. and RIPJ.er W. S., The ARDC Model Atmosphere 1956. Air Force Survey in "Geophysics " 86, Geophysics Research Directorate, Air Force Cambridge Research Center (1956).

(') Centro Studi Propulsione a Reazione, Istituto Nazionale per le Applicazioni di Calcolo del C.N.R., Caratteristiche dell'atmosfera $1 R D C 1956$.

$\left.{ }^{8}\right)$ Aut. diversi, Handbook of Geophysics for Lir Force Designer. Geophysies Research Directorate (1957).

(9) Jastrow R., Results of Experiments in Space. "Journal of the Aerospace Sciences ", 29, 4, (Aprile 1962).

(10) Naporitano L. G., Contributo alla magnetofluidodinamica. "Missili", 1 (1959).

(11) Napolipano L. G., Introduzione allo studio dell'Aerotermochimica. "L'Aerotecnica", XXXVIII, 2, (1958).

${ }^{\left({ }^{2}\right)}$ Napolitano L. G., Moti a simmetria assiale di una miscela di gas a compositione non uniforme. "Aerotecnica", XXXVII, 3, (1957).

${ }^{\left({ }^{13}\right)}$ Napoititano L. G., La Gasdinamica delle miscele nell'approssimazione dello strato limite "L'Aerotecnica", XXXVII, 2, (1957).

${ }^{\left({ }^{14}\right)}$ Pozzi A., Getti di fluidi elettricamente conduttivi in presenza di campi magnetici. "Missili ", 6, (Dicembre 1959).

${ }^{\left({ }^{5}\right)}$ D'ELIA G., Moti Bidimensionali di Gas in presenza di reazioni chimiche, Tesina di laurea (non pubblicata), anno accademico 1957-58, Universita di Napoli.

(16) Minzner R. A., Campion I. S. W., Ponis H. L., The ARDC Model Atmosphere 1959. "Geophysics Research Directorate " Bedford, Massachusetts, (August 1959).

$\left({ }^{17}\right)$ Lewis E. Miluen, MLolecular Weight of Air High Altitudes. "Journal of Geophysical Research ", 62, 3, (September 1957).

${ }^{(18)}$ SnkLavskcy I. S. and U. G. Kunt, The determination of the Density of the Atmosphere at an Altitude of 430 Kilometers by the Sodium Vapor Difjusion Method, NASA Tech. Transl. F 15, (April 1960). 
$\left({ }^{19}\right)$ Townsend J. W. Jr. and Stavin R. M., Aerobe Development Program. "Jet Propulsion", 27, 3 March 1957).

$\left({ }^{20}\right)$ Lestie M. J., W. H. Haussex, N. W. Spexcen, The Nike-Cayum Sounding Rocket. "Jet Propulsion ", 27, 3, (March 1957).

(21) House W. C., Dobge C. H., Future Sounding Rockets, "Jet Propulsion ", 27, 3, (March 1957).

$\left({ }^{22}\right)$ Singer F. S. and Lawrence, A. I., T'errapin an Upper Atmosphere Research Tehicle. "Jet Propulsion", 27, 3, (March 1957).

(23) Davies Halde, General Survey. Proceedings of the Tenth AGARI) (ieneral Assembly, Turkey, 3-7 October 1960.

$\left({ }^{24}\right)$ Stenne T. E., An Atmospheric Model and Some Remarclis on the Interference of Density from the Orbiter of a Close Earth Satellite. "The Astronautical Journal ", 63, 3, Mareh 1958).

${ }^{(25)}$ Berrakov Y. Y. and V. F. Proskunis, Perturbations of Orbites of A rtificial Satellites due to Air Resistance. NASA Technical Translation F. 46, (Nov. 1960).

${ }^{(26)}$ Mikimevicir V. V., B. S. Danilin, A. I. Reprev and L. A. Sokolov, Some Results of the Determination of the Structural Parameters of the itmosphere using the Third Soviet irtificial Earth Satellite. Nasa Tech. Transl. F. 13, (May 1960).

${ }^{(27)}$ Office of Public Information, NASA, Space Activities Summary. 